\title{
An Introduction to Multimedia Technology and Enhanced Learning
}

\author{
Liyun Xia ${ }^{1,2}$ - Shuai Liu ${ }^{2,3,4}$ \\ Accepted: 30 October 2020 / Published online: 6 November 2020 \\ (C) Springer Science+Business Media, LLC, part of Springer Nature 2020
}

\section{Editorial}

Multimedia technology applies interactive computer components, such as graphics, text, video, sound, and animation, to deliver a piece of information $[1,2]$. On the other hand, enhanced learning originated from human education, and now its idea conceptually plays an important role in teaching computers to learn from multimedia data [3-5]. In this way, this issue aims to provide an opportunity for researchers to publish their gifted theoretical and technological studies of advanced multimedia technology and enhanced learning methods, as well as their novel engineering applications within this domain. Finally, this issue received 29 submissions and accepted 10 out of them with at least 2 rounds of strict reviews, with acceptance ratio $34.48 \%$. This editorial is divided into two sections, which aims to the medical image processing methods in the first section, as well as models of real enhanced learning methods in the second section.

\subsection{Medical image processing}

The first section of this issue includes five papers, which focuses on the novel medical image processing methods, such as novel deep learning models, filters and classifications [6-10].

Medical image analysis plays an indispensable role in both scientific research and clinical diagnosis. The first article, "A Review of Deep Learning on Medical Image Analysis", authored by Yudong Zhang from the School of Mathematics

Shuai Liu

liushuai@hunnu.edu.cn

1 Hunan Normal University Journals, Hunan Normal University, Changsha, China

2 Hunan Provincial Key Laboratory of Intelligent Computing and Language Information Processing, Hunan Normal University, Changsha, China

3 Hunan Xiangjiang Artificial Intelligence Academy, Changsha, China

4 College of Information Science and Engineering, Hunan Normal University, Changsha, China

and Actuarial Science, University of Leicester, UK, as well as the Department of Information Systems, King Abdulaziz University, Saudi Arabia, provided a survey of deep learning methods for medical image analysis. Their paper focused on transfer learning because it is characterized by simplicity, efficiency and its low training cost, breaking the curse of small datasets. Their study reviewed more than one hundred representative papers from IEEE, Elsevier, Google Scholar, Web of Science and various sources published from 2000 to 2020, and proposed a comprehensive review, including (i) structure of CNN, (ii) background knowledge of transfer learning, (iii) different types of strategies performing transfer learning, (iv) application of transfer learning in various sub-fields of medical image analysis, and (v) discussion on the future prospect of transfer learning in the field of medical image analysis. This survey will be encouraged to make policy positive to the future development of transfer learning in the field of medical image analysis.

In the process of fine segmentation of medical image, the problem of fuzzy features and non convergence of the threshold in the denoising of details such as contour extraction will be appeared. In this way, the second article, "Contour Feature Extraction of Medical Image based on Multi-threshold Optimization", authored by Gautam Srivastava from the Department of Mathematics and Computer Science, Brandon University, Canada, as well as the Research Centre for Interneural Computing, China Medical University, Taiwan, analyzed the influence of contour wave transformation on the gray correlation degree and noise intensity of different medical images by the improved Bayesian threshold. Then, genetic algorithm was used to find the best quasi threshold in search space. Through this value, the attribute histogram of medical image was established, the best feature extraction threshold of medical image was obtained by golden section method, and the contour feature information of medical image was extracted. Experimental results showed that their method achieved the fast extraction of the contour feature information of the running image, got the ideal feature extraction effect with a high efficiency of feature extraction. 
The application of the traditional single frame character image super-resolution reconstruction method has some problems, such as noise is hard to remove completely and antiinterference performance is poor. The third article, "An Image Super-resolution Reconstruction Method with Single Frame Character based on Wavelet Neural Network in Internet of Things", authored by Marcin Woźniak from the Faculty of Applied Mathematics, Silesian University of Technology, Poland, constructed a new method for the super resolution reconstruction of single frame character image based on wavelet neural network. An image degradation model was established to simulate the degradation process of ideal highresolution image to low-resolution image. Wavelet threshold denoising method was used to remove the noise in a single frame character image and improved the anti-interference performance of the proposed method. Experimental results showed that the accuracy of their method was maintained at $80 \% \sim 90 \%$. With the increase of the number of experimental images, the retention rate of image details remained between $80 \%$ and $95 \%$, indicating that the method was effective in practical application.

Feature extraction of medical CT images is a critical issue in image processing since it affects the accuracy of clinical diagnosis, which is also considered as an important issue to detect sports tear injury. The forth article, "Feature Extraction for Medical CT Images of Sports Tear Injury", authored by Jerry Chun-Wei Lin from the Department of Computer Science, Electrical Engineering and Mathematical Sciences, Western Norway University of Applied Sciences, Norway, presented a feature extraction algorithm for medical CT images of sports tear injury. In their designed model, the CT images were firstly decomposed into a low frequency component and a series of high frequency components in different directions with fast wavelet decomposition. Then, the highand low- frequency information of CT images were enhanced by wavelet layered multi-directional image enhancement algorithm, thus the multi-scale enhanced the medical CT images of sports tear injury. Experimental results showed that the proposed algorithm effectively extracts $\mathrm{CT}$ image features of sports tear injury and provides auxiliary information for diagnosis.

The fifth article, "Synthesizing Multi-Contrast MR Images via Novel 3D Conditional Variational AutoEncoding GAN", authored by Shuihua Wang from the School of Architecture Building and Civil engineering, Loughborough University, UK, compared Magnetic Resonance (MR) and Computer Tomography (CT), two different modalities of medical images, which provided mutually-complementary information to doctors in clinical applications. Their research presented a novel generative network, called CAE-ACGAN, which incorporates the advantages of Variational Auto-Encoder (VAE) and
Generative Adversarial Network (GAN) with an auxiliary discriminative classifier network. Their main contributions included: 1) alleviate the problems of images blurriness and mode collapse by integrating the advantages of VAE and GAN; 2) solve the complicated cross-domain, multicontrast MR synthesis task; 3) use random extraction-patches to lower the limit of insufficient training data with promising results. By comparing with other typical networks, their contribution was yielding nearerreal, higher-quality synthetic MR images, demonstrating the effectiveness and stability.

\subsection{Enhanced learning methods in real application}

Then, the second section of this issue includes the other five papers, which focuses on the enhanced learning methods using in real application, such as forecast, localization, as well as various kinds of applilcations [11-15].

Short-term air traffic flow prediction is essential in the field of air traffic flow management. The sixth article, "Research on Air Traffic Flow Forecast based on ELM Non-Iterative Algorithm", authored by Zhaoyue Zhang from the School of Aeronautics, Northwestern Polytechnical University, China, as well as the College of Air Traffic Management, Civil Aviation University of China, China, studied the chaotic characteristics of air traffic flow with ADS-B data easily available to ground aviation users. Then, a high-dimensional prediction model of air traffic flow time series based on the non-iterative PSR-ELM algorithm was established. Moreover, airspace operation data before and after the outbreak of the COVID-19 epidemic were selected as the experimental scene, and the prediction effects of time series with different degrees of chaos were comparatively analyzed. The experimental results revealed that the PSR-ELM algorithm achieved fast and accurate results, and, when the traffic flow state was sparse, the degree of chaos was reduced and the prediction effect is improved.

In order to overcome the uncertainty of the data sampling period of the sensor due to equipment reasons, the seventh article, "Improving Tightly LiDAR/Compass/ Encoder Integrated Mobile Robot Localization with Uncertain Sampling Period Utilizing EFIR Filter", authored by Yuriy S. Shmaliy from the Department of Electronics Engineering, Universidad de Guanajuato, Mexico, developed a mobile robot localization system under the uncertain sampling period for the tightly-fused light detection and ranging (LiDAR), compass, and encoder data. Based on the tightly-integrated nonlinear model, the extended unbiased finite-impulse response (EFIR) filter fused the sensors' data for the integrated localization system. The performances of the traditional loosely coupled integration scheme, tightly-coupled integration 
scheme with a constant sampling interval, and tightlycoupled integration with an uncertain sampling interval were compared based on real data. Experimental results showed that the proposed scheme was more accurate than the traditional loosely-coupled integration and the one relying on a constant sampling interval, which improves by about $10.2 \%$.

The existing building surface data fusion algorithms do not extract the segmented data features, resulting in inaccurate fusion results. Thus, the eighth article, "Dynamic Fusion Algorithm of Building Surface Data in Heterogeneous Environment", authored by Jing Gao from the College of Computer and Information Engineering, Inner Mongolia Agricultural University, China, proposed a clustering fusion algorithm based on mutual information and fractal dimension in heterogeneous environment. The regression coefficient was used to express the sequence, and the data feature representation and data dimension reduction were realized. The piecewise aggregation approximation method was used to segment the data and extract the features for the long dynamic data series. Experimental results showed that the accuracy of building surface data fusion was greatly improved by using the dynamic data fusion algorithm with the highest value 0.98 , and the sum of square error was reduced with the lowest value 90.44 .

In order to improve the search performance of rich text content, the ninth article, "System Design of Cloud Search Engine Based on Rich Text Content", authored by Arun Kumar Sangaiah from the Vellore Institute of Technology, India, as well as the Department of Industrial Engineering and Management, National Yunlin University of Science and Technology, Taiwan, designed a cloud search engine system based on rich text content. Several hardware devices such as Solr index server, collector, Chinese word segmentation device and searcher were installed, and the data interface is adjusted based on traditional hardware system. Then, the open source Apache Tika framework was used to obtain the metadata of rich text documents and segment word according to the rich text content and semantics, and calculate the weight of each keyword. BM25 algorithm was used to calculate the similarity between keywords and text, and output the search results of rich text. Experimental results showed that the proposed system has high recall rate, high throughput, and short construction time.

The tenth article titled "Personalized learning resource recommendation method based on dynamic collaborative filtering", authored by Weina Fu from the Hunan Provincial Key Laboratory of Intelligent Computing and Language Information Processing, China, as well as the Hunan Xiangjiang Artificial Intelligence Academy, China, proposed a personalized learning resource recommendation method based on dynamic collaborative filtering algorithm. Pearson correlation coefficient was used to calculate the data similarity between learning users or project resources in the network. In order to solve the problems of sparse data and poor scalability in collaborative filtering algorithm, dynamic k-nearest-neighbor and Slope One algorithm were introduced. Fuzzy adaptive binary particle swarm optimization was used to solve the optimal sequence recommendation. Experimental results showed that the proposed method had higher matching degree and faster recommendation speed.

Acknowledgements The guest editors are thankful to our reviewers for their effort in reviewing the manuscripts. We also thank the Edit-in-Chief, Dr. Imrich Chlamtac for his supportive guidance during the entire process. The special issue is sponsored by the Natural Science Foundation of Hunan Province under Grant 2020JJ4434, the Key Scientific Research Projects of Department of Education of Hunan Province with No.19A312; Hunan Provincial Science \& Technology Project Foundation (2018TP1018, 2018RS3065), National Natural Science Foundation of China with No. 61502254.

\section{References}

1. Liu S, Wang S, Liu X, Lin CT, Lv Z (2020) Fuzzy detection aided realtime and robust visual tracking under complex environments. IEEE Trans Fuzzy Syst:1. https://doi.org/10.1109/TFUZZ.2020.3006520

2. Liu S, Guo C, Fadi A et al (2020) Reliability of response region: a novel mechanism in visual tracking by edge computing for IIoT environments. Mech Syst Signal Process 138:106537

3. Ke Q, Zhang J, Wozniak M, Wei W (2019) The phase and shiftinvariant feature by adaptive independent subspace analysis for cortical complex cells. Inf Technol Control 48:58-70

4. Lin Y, Li Y, Yin X, Dou Z (2018) Multisensor fault diagnosis modeling based on the evidence theory. IEEE Trans Reliab 67(2):513-521

5. Liu S, Liu D, Srivastava G, et al (2020) Overview of correlation filter based algorithms in object tracking. Complex \& Intelligent Systems. https://doi.org/10.1007/s40747-020-00161-4

6. Wang J, Zhu H, Wang S-H et al (2020) A review of deep learning on medical image analysis. Mob Netw Appl. https://doi.org/10. 1007/s11036-020-01672-7

7. Li W, Huang Q, Srivastava G (2020) Contour feature extraction of medical image based on multi-threshold optimization. Mob Netw Appl. https://doi.org/10.1007/s11036-020-01674-5

8. Guo L-L, Woźniak M (2020) An image super-resolution reconstruction method with single frame character based on wavelet neural network in internet of things. Mob Netw Appl. https://doi.org/ 10.1007/s11036-020-01681-6

9. Nie Q, Zou Y-B, Lin JC-W (2020) Feature extraction for medical ct images of sports tear injury. Mob Netw Appl. https://doi.org/10. 1007/s11036-020-01675-4

10. Yang H, Lu X, Wang S-H, Lu Z, Yao J, Jiang Y, Qian P (2020) Synthesizing multi-contrast MR images via novel 3D conditional Variational auto-encoding GAN. Mob Netw Appl. https://doi.org/ 10.1007/s11036-020-01678-1

11. Zhang Z, Zhang A, Sun C et al (2020) Research on air traffic flow forecast based on ELM non-iterative algorithm. Mob Netw Appl. https://doi.org/10.1007/s11036-020-01679-0

12. Xu Y, Shmaliy YS, Ma W, et al (2020) Improving tightly lidar/ compass/encoder integrated mobile robot localization with uncertain sampling period utilizing efir filter. Mob Netw Appl. https:// doi.org/10.1007/s11036-020-01680-7

13. Zhu J, Gao J (2020) Dynamic fusion algorithm of building surface data in heterogeneous environment. Mob Netw Appl. https://doi. org/10.1007/s11036-020-01677-2

14. Chan H-P, Xu L, Liu H-H et al (2020) System design of cloud search engine based on rich text content. Mob Netw Appl. https:// doi.org/10.1007/s11036-020-01676-3 
15. Wang H, Fu W (2020) Personalized learning resource recommendation method based on dynamic collaborative filtering. Mob Netw Appl. https://doi.org/10.1007/s1 1036-020-01673-6

Publisher's note Springer Nature remains neutral with regard to jurisdictional claims in published maps and institutional affiliations.

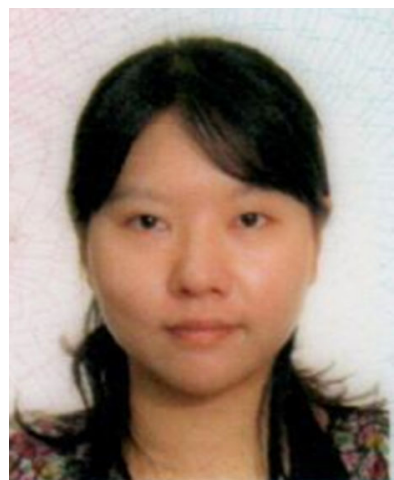

Liyun Xia received her B.A. in Foreign Languages and Literature from Wuhan University in 2005, and received her M.L. in Diplomacy from Peking University in 2008. She is now an editor in Hunan Normal University Journals, Hunan Normal University. Her research interests include educational technology and intelligent education.

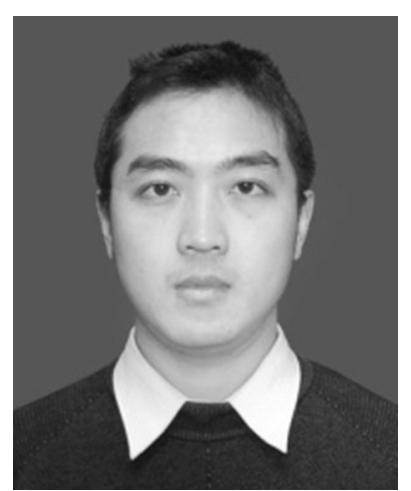

Shuai Liu received his BS, MS and $\mathrm{PhD}$ e from Jilin University in 2002, 2004, and 2011. He used to work as a full professor in College of Computer Science, Inner Mongolia University. Now he acts as a full professor in College of Information Science and Technology, and Vice director of Hunan Provincial Key Laboratory of Intelligent Computing and Language Information Processing, Hunan Normal University. He is now a senior member of China Computer Federation. His research area contains big data processing, computer vision, and information processing. He has published more than 50 papers, with more than 1300 citations. He serves editor role for many respected journals. 